\title{
Breastfeeding and vitamin D supplementation reduce the risk of Kawasaki disease in a German population- based case-control study
}

K. Meyer ${ }^{r^{*}}$ (D, A. Volkmann², M. Hufnagel ${ }^{3}$, E. Schachinger ${ }^{1}$, S. Klau², J. Horstmann ${ }^{1}$, R. Berner ${ }^{4}$, M. Fischer ${ }^{5}$, A. Lehner ${ }^{5}$, N. Haas $^{5}$, S. Ulrich ${ }^{5}$ and A. Jakob ${ }^{5}$

\begin{abstract}
Background: In Kawasaki disease (KD), a vasculitis of unknown etiology, the most serious complication is the development of coronary artery aneurysm (CAA). To date, the exact pathomechanism of KD is unknown. Both environmental and genetic factors seem to be associated with the development of the disease.

Methods: Data on KD patients recruited from the population-based German Pediatric Surveillance Study during 2012-2014 were used to evaluate the impact of various factors from the perinatal and infancy period on the development of KD. The study design was a matched case-control study with respect to age, sex and place of residence ( $n=308 \mathrm{KD}$ cases, $n=326$ controls). All KD patients were individually re-evaluated; all fulfilled the international diagnostic KD criteria. A standardized questionnaire was used to review breastfeeding practices, vitamin D supplementation and birth characteristics. Logistic regression analyses were performed to obtain odds ratios (OR) for various risk factors among the case-control pairs. Simple measures of association were used to assess the impact of these factors on the clinical course.

Results: There was no difference in lengths of gestation, birth weight or parturition between KD patients and controls, but independently from each other vitamin D supplementation and breastfeeding were negatively associated with KD, even when adjusted for age, place of residence and sex. The duration of vitamin $D$ was significantly shorter among children with KD than among children without KD ( $p=0.039$, OR $=0.964,95 \%$ Cl: $0.931-$ 0.998 ), as was the duration of breastfeeding ( $p=0.013, \mathrm{OR}=0.471,95 \% \mathrm{Cl}: 0.260-0.853)$. Comparing KD patients with and without breastfeeding and/or vitamin $D$ supplementation, there were no differences regarding developing CAA, being refractory to intravenous immunoglobulin treatment, age at onset of the disease and levels of inflammatory laboratory values.
\end{abstract}

Conclusion: Our findings indicate breastfeeding and vitamin D supplementation to have protective effects in association with KD in our study population; however, these seem not to influence the natural course of the disease. Although the overall effects were relatively small, they nevertheless underline the overall benefit of both interventions.

Trial registration: Clinical Trial Registration: German clinical trial registration, http://apps.who.int/trialsearch/Trial2. aspx?TriallD=DRKS00010071. Date of registration was 26. February 2016. The trial was registered retrospectively.

Keywords: Kawasaki disease, Coronary artery aneurysm, Risk factors, Vitamin D supplementation, Breast feeding

\footnotetext{
* Correspondence: karomeyer@hotmail.com

'Department of Congenital Heart Disease and Pediatric Cardiology,

University Heart Center Freiburg, Mathildenstraße 1, D-79106 Freiburg,

Germany

Full list of author information is available at the end of the article
}

(c) The Author(s). 2019 Open Access This article is distributed under the terms of the Creative Commons Attribution 4.0 International License (http://creativecommons.org/licenses/by/4.0/), which permits unrestricted use, distribution, and reproduction in any medium, provided you give appropriate credit to the original author(s) and the source, provide a link to the Creative Commons license, and indicate if changes were made. The Creative Commons Public Domain Dedication waiver (http://creativecommons.org/publicdomain/zero/1.0/) applies to the data made available in this article, unless otherwise stated. 


\section{Background}

Kawasaki disease (KD) is a vasculitis of unknown etiology, which can be complicated by the development of coronary artery aneurysm (CAA). It is a rare disease, primarily affecting children under five years of age. The incidence among Asian children is much higher than among Caucasians. In our 2011-2012 cohort, we estimated an incidence of 7.2/100,000 in Germany (children $<5$ years) [1, 2]. By contrast, for the year 2012 Japan reported the highest incidence worldwide, with 265/ 100,000 children (aged 0-4 years) diagnosed [3]. Cause and pathogenesis of $\mathrm{KD}$ are unclear. A combination of different risk factors, for disease development, along with a genetic predisposition, so far has been assumed $[2,4,5]$. To further investigate this assumption, we analysed the impact of different risk factors for KD during the perinatal period and infancy. Week of gestation, birth weight and type of parturition are known to impact health in later life [6]. Caesarean delivery, for example, is known to be a risk factor for multiple chronic immune diseases, including asthma and juvenile idiopathic arthritis [7]. Similarly, vitamin D supplementation and breastfeeding are well-known for their health benefits: In Germany, vitamin D supplementation generally is recommended for the first year of life. Besides playing an important role in the bone and calcium balance [8], vitamin D also regulates other cell functions and supports the immune system overall [9]. Furthermore, epidemiological studies on vitamin D deficiency show potential associations with cardiovascular diseases [10] and vasculitis [11].

For its health and other benefits, breastfeeding is preferable to formula feeding. Among other advantages, breastfeeding seems to protect against a variety of infectious diseases [12] and non-breastfed children have a higher risk of developing allergies and bronchial asthma later in life [13]. A Japanese study already has indicated a potential protective effect in KD [14].

During the perinatal and infancy periods, these and other factors potentially play a role in the pathogenesis of KD. Therefore, in a population-based, retrospective case-control study we investigated the association and impact of birth characteristics, breastfeeding practices and vitamin D supplementation on KD.

\section{Methods}

Our study was designed as an epidemiological case-control study. KD cases were recruited from the population-based German Pediatric Surveillance Study (ESPED) from January 2012 to December 2014 [15]. For the ESPED report, pseudonymous identifiers were used. A standardized questionnaire was sent to reporting physicians requesting clinical and laboratory details from each KD case. Additionally, we requested that reporting physicians ask parents (via written informed consent) to agree to disclosure of their child's identity so that our group could conduct follow-up questioning. In cases where such consent was provided, it became possible for us to validate the original ESPED data via discharge letters, recorded laboratory values and echocardiographic findings. For details see Additional file 1.

Due to data protection regulations, we only were able to include those KD cases where parents had provided written informed consent. Data regarding breastfeeding practices, vitamin D supplementation and birth characteristics were collected via a standardized questionnaire, and responses were provided online and in telephone interviews (see Additional file 2).

In order to obtain control cases from the KD patient's own immediate environment, control cases $(n=326)$ were recruited from among the personal contacts of the KD case. First, the parents of KD cases were asked whether they might be able to recruit one of their child's friends to take part in the survey. If no immediate friend of the KD cases was available or interested, then the treating paediatrician was asked to find a child who met the following criteria: (1) same sex, (2) same place of residence ( $\leq 50 \mathrm{~km}$ distance), (3) same age ( $\leq 6$ months age difference) and (4) no medical history of KD. Once KD and control cases had been established, data were collected in an anonymous way through an online questionnaire. Case-control-pairing was conducted by using the ESPED pseudonymous identifier. In some cases, more than one control case was able to be recruited. For these KD cases, the control case was selected manually accordingly to best fit, which was based upon: (1) age, (2) gender and (3) place of residence. The best fitting case-control-pair was used for the statistical analysis.

All ESPED-reported KD cases $(n=631)$ were re-evaluated and checked for fulfilling the American Heart Association (AHA) guidelines for classification as either complete or incomplete KD cases [16]: Complete cases were defined as those with persistent fever for $\geq 5$ days or fever that resolved within five days in response to intravenous immunoglobulin (IVIG) treatment. In addition, display of at least four principal clinical features was required: (1) changes in extremities; (2) polymorphous exanthema; (3) bilateral conjunctival injection without exudate; (4) enanthema of lips and oral cavity; and (5) bilateral cervical lymphadenopathy. By contrast, incomplete cases included either (A), (B) or (C) as outlined below:

(A) Those with fever (independent of age), plus fewer than four clinical features, plus detection of CAA. Diagnosis of CAA was based upon the clinical judgment of the reporting physician. In Germany, two criteria for CAA are applied: The first criterion 
used is from the Japanese Ministry of Health, which defines aneurysms either as a lumen $>3 \mathrm{~mm}$ in children under 5 years old, or as a diameter 1.5 times the size of the surrounding segment, or else as a clearly irregular lumen. The second criterion applied is a Z-score of above 2.5 for one of the coronary arteries.

(B) Those with fever under 6 months of age who have fewer than four clinical features.

(C) Those with fever over 6 months of age, plus three clinical features, plus laboratory evidence of systemic inflammation (CRP $\geq 30 \mathrm{mg} / \mathrm{dl}$ or ESR $\geq 40$ $\mathrm{mm} / \mathrm{h}$ ) in combination with at least three of the following other abnormal supplemental laboratory findings: (1) increased alanine transaminase, (2) albumin $\leq 3.0 \mathrm{~g} / \mathrm{dL}$, (3) leukocyturia, (4) anaemia for age, (5) leukocytosis $\left(\geq 15,000 / \mathrm{mm}^{3}\right)$ and (6) thrombocytosis $\left(\geq 450,000 / \mathrm{mm}^{3}\right)[16]$.

In accordance with AHA guidelines, in cases where fever persists for over $36 \mathrm{~h}$, German recommendations stipulate the administration of a second IVIG therapy course. Our study defined such cases as refractory to IVIG.

Out of a total of $n=631$ reported KD cases, $n=69$ cases did not fulfil the AHA case definitions. Follow-up with complete data in response to our questionnaire was available in $n=308$ cases. For details see Fig 1 .

\section{Statistics}

First, we analysed the quality of our matching (depending on the factors: age, place of residence and sex). Cross-tables, measures of association (phi coefficient, contingency coefficient, Cramer's V and Pearson correlation coefficient) and OR were used. To identify possible risk factors for $\mathrm{KD}$, we performed univariate analyses. For the main analysis, we employed a logistic regression conditional on the case-control pairs. As a sensitivity analysis, we also applied an unconditional logistic regression adjusting for the matching factors. The latter approach uses all available observations without restriction on successfully matched cases and controls; this results in a higher statistical power but can introduce some bias [17]. Therefore, the results of the conditional logistic regression were taken to represent our main results, while the results of the unconditional logistical regression were used as a sensitivity analysis to verify the main results.

All potential risk factors were analysed for associations with clinical findings (developing CAA, level of inflammatory laboratory values, age at onset of disease and being refractory to IVIG treatment). For this, we used the Chi-squared test, Spearman's rank correlation coefficient and Cramer's V. For breastfeeding and vitamin D supplementation, the age at onset of disease was examined

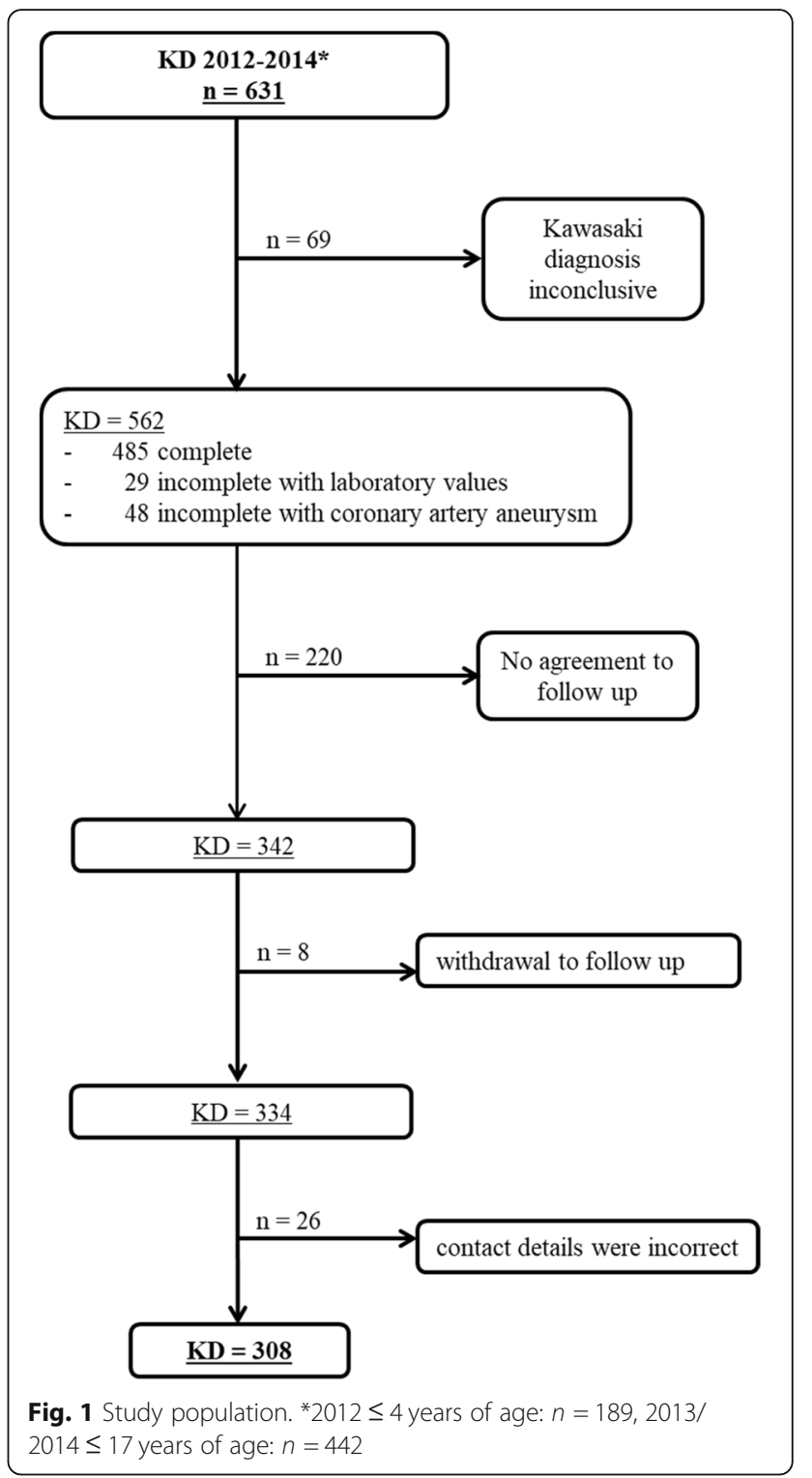

using the Mann-Whitney test. Statistical analyses were performed using the statistical software $\mathrm{R}$ (3.3.2) and SPSS (24.0.0.0, IBM).

\section{Matching}

A case-control matching was made based on sex, age and place of residence (Figs. 2, 3 and Table 1). The measures of association, i.e. contingency coefficient and Cramer's $\mathrm{V}$ were $>0.9$ for age and place of residence. This attests to the high quality of the matching and furthermore shows that the results would have been distorted, had the matching been ignored. With regard to sex, the measures of association were between 0.577 and 0.706 , indicating a satisfactory case-control matching. Here, the OR for the matching factor sex was 36,346, meaning that the likelihood that a KD and control case would have 


\section{Matching for Age at Onset of Fever in Cases}

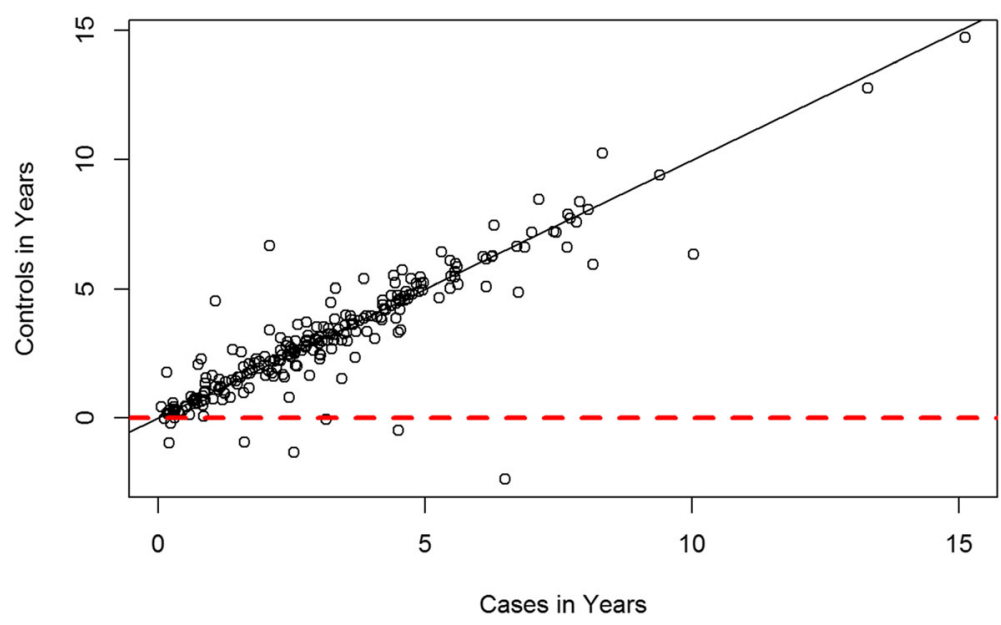

Fig. 2 Scatterplot - Matching KD and control cases by age at the onset of fever in KD cases. One dot meets a matched pair. Dots that are below the red line were excluded. It is noticeable that most dots are on or next to the diagonal. Consequently, a successful matching based on age can be assumed

the same sex was 36 times higher than that of their having different sexes.

\section{Results}

Our study cohort was composed of $n=308 \mathrm{KD}$ cases and $n=326$ controls (Fig. 1). The median age of KD cases during the acute phase of the disease was 30 months. On the day that the data collection was completed (March 22, 2017), the median age of KD cases was 6.5 years, and $63 \%$ were male. Control cases had a median age of 6.3 years on the day of the data collection, and $58 \%$ were male (Table 2). KD and control cases were reported from all regions of Germany (Fig. 3). Ethnic background was available from $95.1 \%$ of KD cases $(n=$ 293) and showed a breakdown of $97.6 \%$ Caucasian $(n=$ $286), 1.7 \%$ Asian $(n=5)$ and $0.7 \%$ black African $(\mathrm{n}=2)$.

\section{Perinatal risk factors compared in KD cases vs. control cases}

The dataset matching was prepared for optimal comparison. Thus, only complete case-control pairs (maximum $n=227$ ) were used for the conditional logistic regression. When information was missing for one risk factor, the case-control pair was excluded from these analyses. As a sensitivity analysis, we also applied the unconditional logistic regression (maximum $n=542$ ). In cases where the results differed from those of the conditional logistic regression, we discussed the discrepancy. For simple description, the entire data set $(n=308 \mathrm{KD}$ cases and $n=326$ control cases) was taken into consideration.

Among the perinatal factors, there were no significant differences between $\mathrm{KD}$ and control cases regarding week of gestation, birth weight and parturition (Table 3). However, breastfeeding and vitamin D supplementation did show a protective effect for KD (Table 3). The OR for breastfeeding for longer than two weeks was 0.471 [95\% CI 0.260-0.853] as compared to breastfeeding for less than two weeks.

The evaluation of vitamin D supplementation also revealed small but significant differences between the $\mathrm{KD}$ group and the control group. Our analysis showed that KD cases received Vitamin D for significantly shorter time periods than did the controls (Mean cases $=5$ months versus Mean controls $=6$ months, OR 0.96, 95\% CI 0.931-0.998, $p=0.039$, see Table 3).

To estimate a possible confounding, the association between breastfeeding and vitamin $\mathrm{D}$ supplementation was tested. The measurements of association showed no obvious associations between vitamin $\mathrm{D}$ supplementation and breastfeeding: Spearman-Rang-Correlation $\left(\mathrm{r}_{\mathrm{s}}=\right.$ - 0.039), Eta-Squared $\left(\eta^{2}=0.000\right)$ and Cramer's V $(\mathrm{V}=$ $0.141)$.

\section{Influence of risk factors on the clinical course}

We tested whether the duration of breastfeeding and of vitamin D supplementation influenced the clinical course of KD cases. The clinical variables CAA, being refractory to IVIG treatment, and the level of inflammatory serum markers were analysed to compare KD cases with shorter vs. longer breastfeeding periods and/or longer vs. shorter vitamin D supplementation. Comparing $\mathrm{KD}$ cases with CAA (in the acute phase) to KD cases without detection of CAA did not show significant differences with respect to the duration of breastfeeding and vitamin D supplementation. Similarly, KD cases 


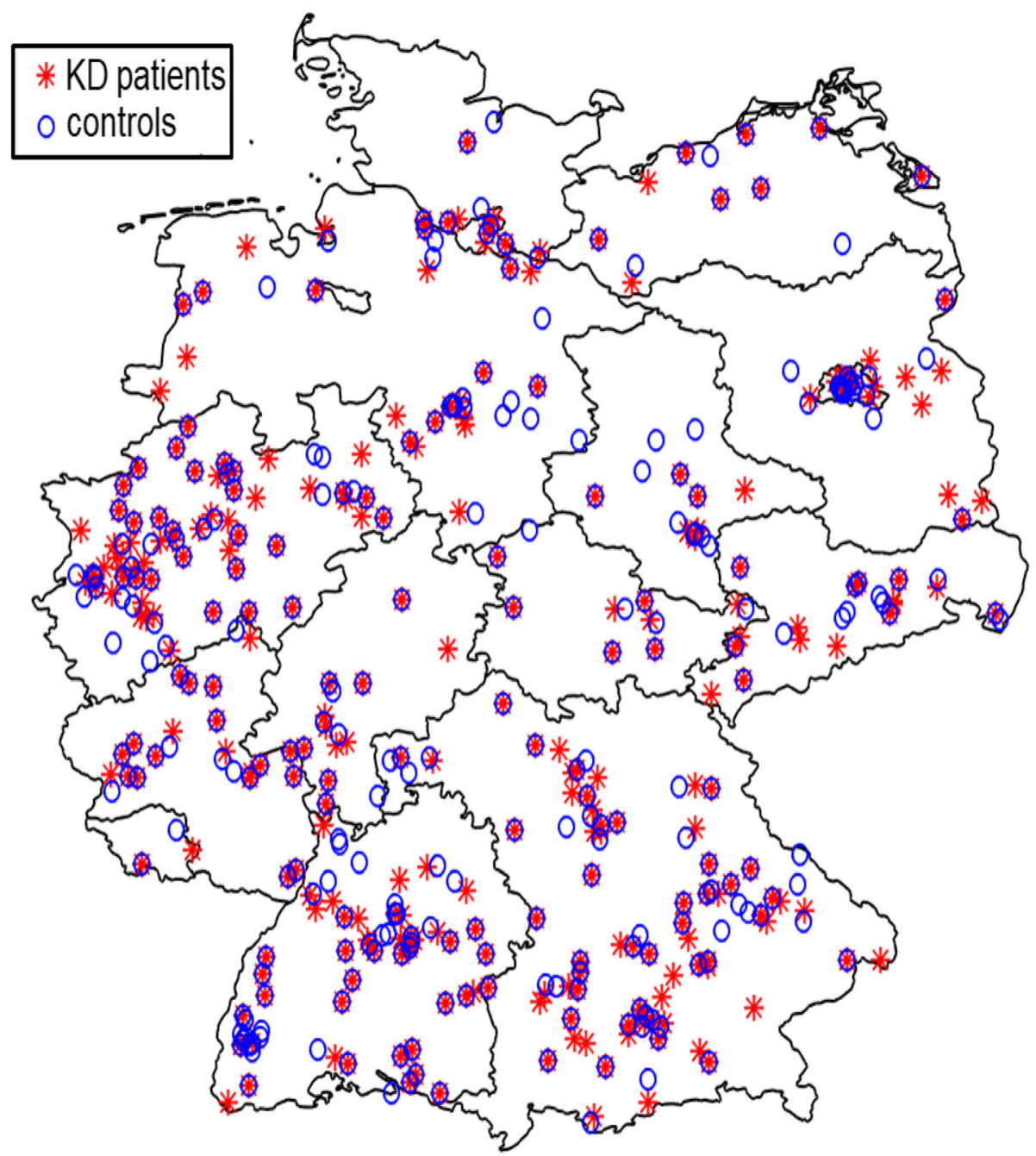

Fig. 3 Matching: Geographical distribution of KD patients and their controls within Germany. We have created a map of Germany with the software MATLAB. Shapefiles, a file format containing geodata from the Database of Global Administrative Areas were used to extract the administrative boundaries in Germany. The individual postcodes were assigned to the respective federal state

being refractory to IVIG did not differ from IVIG responders (Table 4). Also, the impact on systemic inflammatory serum markers (CRP, leukocytes and platelets) did not demonstrate any significant difference (Table 4).

In addition, we investigated whether breastfeeding and vitamin D supplementation might influence disease

Table 1 Measures of association for the matching factors sex, age and place of residence

\begin{tabular}{llll}
\hline & \multicolumn{3}{l}{ Matching factors } \\
\cline { 2 - 4 } & Sex & Age & Place of residence \\
\hline Measures of association & & & \\
Phi coefficient & 0.706 & & \\
Contingency coefficient & 0.577 & 0.945 & 0.945 \\
Cramer's V & 0.706 & 0.961 & 0.961 \\
Pearson correlation coefficient & & 0.901 & \\
\hline
\end{tabular}

onset, since not-breastfed/not-supplemented cases potentially might develop KD earlier than breastfed/supplemented cases (Table 5). However, the median age in the two categories $(<2$ weeks vs. $>2$ weeks of breastfeeding, along with regular vs. irregular supplementation of vitamin D) was not significantly different ( $p$-value 0.802 and 0.534 , respectively). For the duration of vitamin D supplementation, the correlation coefficient was small and negative. The coefficient of the linear model showed no significant relation to disease onset (Table 5).

\section{Discussion}

Our study is the first of a nationwide, population-based cohort in Germany. The study investigates various perinatal and infancy factors in association with Kawasaki disease and its disease course. Our data reinforce the findings of a large study from Japan which has indicated 
Table 2 Clinical details of KD vs. control cases

\begin{tabular}{|c|c|c|}
\hline & $\begin{array}{l}\text { KD cases } \\
(n=308)\end{array}$ & $\begin{array}{l}\text { Control cases } \\
(n=326)\end{array}$ \\
\hline \multicolumn{3}{|l|}{ Age in years ${ }^{a}$} \\
\hline Median & 6.5 & 6.3 \\
\hline \multicolumn{3}{|l|}{ Sex, n (\%) } \\
\hline Female & $114(37)$ & $137(42)$ \\
\hline Male & $194(63)$ & $189(58)$ \\
\hline \multicolumn{3}{|l|}{ KD diagnosis, n (\%) } \\
\hline Complete & 269 & n.a. \\
\hline Incomplete with laboratory changes ${ }^{b}$ & 15 & n.a. \\
\hline Incomplete with CAA & 24 & n.a. \\
\hline \multicolumn{3}{|l|}{ Days until start of IVIG therapy } \\
\hline Average & 6.6 & n.a. \\
\hline Min - max & $0-32$ & n.a. \\
\hline \multicolumn{3}{|l|}{ Refractory to IVIG treatment ${ }^{c}$} \\
\hline Yes $>1$ IVIG cycle & $29(13.4 \%)$ & n.a. \\
\hline \multicolumn{3}{|l|}{ Therapy with steroids, n (\%) } \\
\hline Yes, for KD treatment & $53(17.2)$ & n.a. \\
\hline Yes, for other reasons & $9(2.9)$ & n.a. \\
\hline \multicolumn{3}{|l|}{ CAA in acute phase, $n(\%)$} \\
\hline Yes & $36(11.6)$ & n.a. \\
\hline \multicolumn{3}{|l|}{ CAA after one year ${ }^{c}, \mathrm{n}(\%)$} \\
\hline Yes & $13(5.9)$ & n.a. \\
\hline
\end{tabular}

at the end of the case-control study survey (i.e, March 22, 2017)

${ }^{b}$ according to the guidelines of the American Heart Association at least three other abnormal supplemental laboratory findings, namely (1) increased alanine transaminase, (2) albumin $\leq 3.0 \mathrm{~g} / \mathrm{dL}$, (3) leukocyturia, (4) anaemia for age, (5) leukocytosis $\left(\geq 15,000 / \mathrm{mm}^{3}\right)$ (6) thrombocytosis $\left(\geq 450,000 / \mathrm{mm}^{3}\right)[16]$

'for cases reported in 2013/2014 $n=217$; CAA = coronary artery aneurysma; n.a. $=$ not applicable

that breastfeeding may be protective for KD [14]. Vitamin $\mathrm{D}$ supplementation appears to have a minor protective effect for KD as well. However, an influence of these factors on the clinical course (development of CAA, raised systemic inflammatory markers, being refractory to IVIG treatment) was not observed. Since the risk factors age, sex and place of residence were used as matching factors, they could not be included in our analysis of potential risk factors.

Our data regarding vitamin D supplementation showed that KD cases in our cohort received significantly less vitamin D than their healthy controls. In a recently published study that measured vitamin D levels during the acute phase of KD, levels proved to be significantly lower compared to controls [18]. Stagi et al. also have described a reduced serum vitamin D concentration in KD cases. These authors suggested that vitamin $\mathrm{D}$ may be reduced by the inflammatory process [19]. Our findings indicate that lower vitamin D levels may already play a role before disease onset, since in our cohort, children with KD had a shorter duration of vitamin D supplementation. However, due to our study design, we were not able to determine the actual vitamin D levels prior to the development of KD.

Because the skin of infants is not able to produce sufficient vitamin $\mathrm{D}$, without supplementation, infants will develop vitamin D deficiency [20]. For this reason, in countries such as the USA, infant food is supplemented with vitamin D. In countries such as Germany, a daily vitamin $\mathrm{D}$ supplementation is recommended during the first year of life [8]. The Nutrition Commission of the German Society for Pediatric and Adolescent Medicine recommends 400-500 IU / day during the first year of life [21].

The anti-inflammatory and immunomodulatory effects of vitamin D are well-described in several studies [22]. Vitamin D appears to block factors (e.g., tumor necrosis factor $\alpha$ ) that are essential for the activation of proinflammatory cytokines [23]. This raises the possibility of vitamin $\mathrm{D}$ being used as complementary therapy in immune-mediated diseases such as KD. Some studies already indicate that reduced vitamin $\mathrm{D}$ levels may negatively effect the clinical course. First, reduced vitamin D levels seem to be associated with the prevalence of cardiovascular diseases and the development of CAA [19, 24]. Low vitamin D levels inducing endothelial dysfunction may partially explain this association [25]. Second, Zhang et al. have described significantly reduced vitamin $\mathrm{D}$ levels in KD patients who were refractory to IVIG treatment [18]. We investigated both of these factors CAA development and being refractory to IVIG treatment - but were not able to find a significant association with vitamin D supplementation. Furthermore, due to the anti-inflammatory activity of vitamin $\mathrm{D}$, we also investigated other inflammatory serum markers, including CRP, thrombocytes and leukocytes - ones that might indicate higher disease activity in KD cases. However, the markers did not differ between KD cases with longer vs. shorter vitamin D supplementation. Stagi et al. have reported on a negative association regarding low vitamin D serum levels with CRP titers [19]. Additionally, Jun et al. retrospectively reviewed whether low vitamin D levels were associated with resistance to IVIG therapy in KD cases. They found vitamin D deficiency to be associated with IVIG resistance, but not associated with inflammatory markers [26]. In summary, we conclude that vitamin D could potentially play a role in the inflammatory process of KD. However, for a better understanding of its mechanism, additional prospective studies would be necessary.

The perinatal factor of breastfeeding showed a significant protective effect. In general, breastfeeding is recommended for at least six months due to its various health benefits [27]. Breast milk contains lactoferrin and 
Table 3 Demographic characteristics of KD and control cases and potential perinatal factors for KD development (frequencies, means and logistic regression with $p$-value, OR and 95\% Cl)

\begin{tabular}{|c|c|c|c|c|}
\hline & \multirow[t]{2}{*}{$\begin{array}{l}\text { KD cases } \\
(n=308)\end{array}$} & \multirow[t]{2}{*}{$\begin{array}{l}\text { Control cases } \\
(n=326)\end{array}$} & \multicolumn{2}{|c|}{$\begin{array}{l}\text { Conditional logit }- \text { model }(n=454) \\
\text { Unconditional logit - model }(n=542)\end{array}$} \\
\hline & & & OR [95\% Cl] & $p$-value \\
\hline \multicolumn{5}{|c|}{ Duration of pregnancy in weeks } \\
\hline Average $( \pm \mathrm{SD})$ & $39( \pm 2.057)$ & $39( \pm 1.944)$ & $\begin{array}{l}0.992[0.891-1.105] \\
0.965[0.883-1.050]\end{array}$ & $\begin{array}{l}0.891 \\
0.414\end{array}$ \\
\hline \multicolumn{5}{|l|}{ Parturition ${ }^{a}, \mathrm{n}(\%)$} \\
\hline Vaginal & $128(65.6)$ & $194(70.8)$ & 1 (reference) & \multirow{2}{*}{$\begin{array}{l}0.388 \\
0.302\end{array}$} \\
\hline Cesarean section & $67(34.4)$ & $80(29.2)$ & $\begin{array}{l}1.286[0.727-2.274] \\
1.259[0.813-1.953]\end{array}$ & \\
\hline \multicolumn{5}{|l|}{ Birth weight in gram } \\
\hline Average $( \pm \mathrm{SD})$ & $3340( \pm 527.052)$ & $3383( \pm 537.734)$ & $\begin{array}{l}0.999[0.999-1.000] \\
0.999[0.999-1.000]\end{array}$ & \multirow[t]{2}{*}{$\begin{array}{l}0.715 \\
0.399\end{array}$} \\
\hline Range & $1560-4960$ & $1171-4910$ & & \\
\hline \multicolumn{5}{|c|}{ Duration of breastfeeding, $\mathrm{n}(\%)$} \\
\hline$<2$ weeks & $66(21.4)$ & $30(9.2)$ & 1 (reference) & \multirow{2}{*}{$\begin{array}{l}0.013 \\
0.001\end{array}$} \\
\hline$>2$ weeks $^{b}$ & $242(78.6)$ & $296(90.8)$ & $\begin{array}{l}0.471[0.260-0.853] \\
0.415[0.243-0.687]\end{array}$ & \\
\hline \multicolumn{5}{|c|}{ Vitamin D supplementation in the first year of life, $n(\%)$} \\
\hline No & $64(20.8)$ & $53(16.3)$ & 1 (reference) & \multirow{2}{*}{$\begin{array}{l}0.782 \\
0.051\end{array}$} \\
\hline Yes & $244(79.2)$ & $273(83.7)$ & $\begin{array}{l}0.926[0.537-1.595] \\
0.623[0.383-0.995]\end{array}$ & \\
\hline \multicolumn{5}{|c|}{ Duration of vitamin D supplementation in the first year of life in months } \\
\hline Mean $( \pm S D)$ & $5.16( \pm 5.749)$ & $6.03( \pm 5.596)$ & $\begin{array}{l}0.964[0.931-0.998] \\
0.965[0.935-0.995]\end{array}$ & $\begin{array}{l}0.039 \\
0.024\end{array}$ \\
\hline \multicolumn{5}{|c|}{ Regularity of vitamin D supplementationc, n (\%) } \\
\hline Irregular & $65(26.6)$ & $55(20.0)$ & 1 (reference) & \multirow{2}{*}{$\begin{array}{l}0.042 \\
0.060\end{array}$} \\
\hline Regular & $179(73.4)$ & $220(80.0)$ & $\begin{array}{l}0.559[0.319-0.980] \\
0.642[0.402-1.016]\end{array}$ & \\
\hline
\end{tabular}

${ }^{\mathrm{a}}$ not asked in $n=52$ control cases vs. $n=113 \mathrm{KD}$ cases, ${ }^{\mathrm{b}}$ includes partially breastfed children. ${ }^{c}$ refers only to cases that received vitamin $\mathrm{D}$

lysozyme, which non-specifically inhibit all types of pathogenic microorganism [28]. In addition, secretory immunoglobulin A confers specific protection against mucosal pathogens $[29,30]$. Breastfeeding rarely has been considered in association with KD. Data from a recent study in Japan showed that children who were breastfed were less likely to develop KD [14]. The Japanese authors described a protective effect for both exclusive (OR $0.26 ; 95 \%$ CI $0.12-0.55$ ) and partial breastfeeding (OR 0.27; 95\% CI 0.13-0.55) as compared to formula milk [14]. Even partially breastfed children showed a general benefit. Therefore, the protective effect of breast milk probably is not linked to the duration of breastfeeding but rather to substances in either breast milk or colostrum [14], the latter of which contains high levels of secretory antibodies [31]. Additional investigations will be needed to identify these protective substances. In the future, infants who cannot be breastfed might be able to receive these protective substances for protective purposes.
In general, breastfeeding has a positive effect on the outcome of infections in infants. For example, Nishimura et al. have described shorter hospital stays and better outcomes in breastfed children infected with respiratory syncytial virus [32]. However, the effect of breastfeeding on the outcome of KD remains unknown. In our study, we were unable to find an association between the duration of breastfeeding and the appearance of CAA, being refractory to IVIG treatment or the amount of inflammatory serum markers. Therefore, prolonged breastfeeding seems not to be associated with a milder clinical course in KD. Protection against infection by breast milk, the so-called maternal passive immunity, relates to the first six months of feeding [33]. We investigated the potential impact of breastfeeding and vitamin D supplementation on the age of disease onset. However, in shorter-breastfed and/or shorter-supplemented cases, KD did not occur sooner than in other KD cases. It is therefore questionable whether maternal passive immunity has an effect on the age of KD onset. 
Table 4 Influence of vitamin D supplementation and breastfeeding on the course of disease

\begin{tabular}{|c|c|c|c|c|c|c|}
\hline & \multicolumn{3}{|c|}{$\begin{array}{l}\text { Vitamin D supplementation } \\
(n=308)\end{array}$} & \multicolumn{3}{|c|}{$\begin{array}{l}\text { Breastfeeding } \\
(n=308)\end{array}$} \\
\hline & $0-6$ months & $7-12$ months & $\begin{array}{l}p \text {-value } \\
{[95 \% \mathrm{Cl}]}\end{array}$ & $<2$ weeks & $>2$ weeks & $\begin{array}{l}p \text {-value } \\
{[95 \% \mathrm{Cl}]}\end{array}$ \\
\hline \multicolumn{7}{|l|}{ CAA, n (\%) } \\
\hline Yes & $13(11.4)$ & $23(11.9)$ & \multirow{2}{*}{$\begin{array}{l}0.905 \\
{[-0.079-0.070]}\end{array}$} & $5(7.6)$ & $31(12.8)$ & \multirow{2}{*}{$\begin{array}{l}0.185 \\
{[-0.130-0.025]}\end{array}$} \\
\hline No & 101 (88.6) & $171(88.1)$ & & $61(92.4)$ & $211(87.2)$ & \\
\hline \multicolumn{7}{|c|}{ Refractory to IVIG a n (\%) } \\
\hline Yes & $9(11.5)$ & $20(14.5)$ & \multirow{2}{*}{$\begin{array}{l}0.543 \\
{[-0.125-0.066]}\end{array}$} & $4(8.7)$ & $25(14.7)$ & \multirow{2}{*}{$\begin{array}{l}0.233 \\
{[-0.160-0.039]}\end{array}$} \\
\hline No & $69(88.5)$ & $119(85.5)$ & & $42(91.3)$ & $145(85.3)$ & \\
\hline \multicolumn{7}{|c|}{ Laboratory, average $( \pm S D)$} \\
\hline $\mathrm{CRP}^{\mathrm{b}}$ & $\begin{array}{l}93,55 \\
( \pm 86.703)\end{array}$ & $\begin{array}{l}105,08 \\
( \pm 82.948)\end{array}$ & $\begin{array}{l}0,248 \\
{[-31.119-8.057]}\end{array}$ & $\begin{array}{l}103,04 \\
( \pm 84.499)\end{array}$ & $\begin{array}{l}100,20 \\
( \pm 84.539)\end{array}$ & $\begin{array}{l}0,809 \\
{[-20.255-25.942]}\end{array}$ \\
\hline Thrombocytes $^{c}$ & $\begin{array}{l}235.48 \\
( \pm 280.350)\end{array}$ & $\begin{array}{l}210.95 \\
( \pm 241.805)\end{array}$ & $\begin{array}{l}0.421 \\
{[-35.367-84.431]}\end{array}$ & $\begin{array}{l}570.56 \\
( \pm 277.776)\end{array}$ & $\begin{array}{l}577.64 \\
( \pm 235.789)\end{array}$ & $\begin{array}{l}0.705 \\
{[-54.229-80.070]}\end{array}$ \\
\hline Leukocytes $^{d}$ & $\begin{array}{l}17.44 \\
( \pm 7.853)\end{array}$ & $\begin{array}{l}17.31 \\
( \pm 7.613)\end{array}$ & $\begin{array}{l}0.885 \\
{[-1.667-1.931]}\end{array}$ & $\begin{array}{l}17.23 \\
( \pm 6.582)\end{array}$ & $\begin{array}{l}17.39 \\
( \pm 7.978)\end{array}$ & $\begin{array}{l}0.883 \\
{[-2.279-1.962]}\end{array}$ \\
\hline
\end{tabular}

${ }^{\mathrm{a}}$ only KD patients from $2013 / 2014 \mathrm{n}=217{ }^{\mathrm{b}}{ }^{\mathrm{in}} \mathrm{mg} / \mathrm{dl}^{\mathrm{c}}{ }^{\mathrm{c}}$ in $\mathrm{T} / \mu \mathrm{ll}{ }^{\mathrm{d}}$ in $\mathrm{T} / \mu \mathrm{l}$

In conclusion, breastfeeding seems to have a protective effect on the development of KD, supporting the health benefit of general breastfeeding recommendations.

The strength of our case-control study is based upon the large number of $\mathrm{KD}$ cases in a Germany-wide cohort, along with the excellent comparability between our $\mathrm{KD}$ and control groups. KD cases were reported from all regions of Germany, as were the control cases who were recruited in parallel. A possible bias by the confounder age, sex and place of residence was able to be minimized through a thorough matching process [34]. In addition, all KD cases were evaluated using a standardized questionnaire and they fulfilled internationally-accepted KD criteria [16]. These strict criteria minimized the risk of misdiagnosed KD cases being included in our study. However, the validity of our results should be interpreted in light of our retrospective study design and considered in context of its known limitations. Although $\mathrm{KD}$ cases have been reported as part of a prospective surveillance study, data analysis for this study was based upon a retrospective survey conducted over a period of three years. The time period between the acute phase of KD and that of this survey was at least one year. Data quality strongly depends upon the memory ability of the parents, the so-called recall bias. Therefore, we cannot rule out the possibility that families of sick children may have been more likely to remember potential risk factors and/or living conditions than families in the control group did. Another limitation relates to the fact that KD cases were reported from a large number of hospitals, most of whom were without a standardized treatment protocol. KD therapy varies considerably. Some of our $\mathrm{KD}$ cases received corticosteroids early in the course of disease that might have impacted the patients' clinical

Table $\mathbf{5}$ Influence of vitamin D supplementation and breastfeeding on disease onset

\begin{tabular}{|c|c|c|c|}
\hline & $\begin{array}{l}\text { Age in months }{ }^{a}( \pm S D) \\
\left(n=308^{b}\right)\end{array}$ & $\begin{array}{l}\text { Wilcoxon rank } \\
\text { p-value }\end{array}$ & Spearman correlation coefficient \\
\hline \multicolumn{4}{|c|}{ Regularity of vitamin D supplementation ${ }^{c}$} \\
\hline Irregular & $34.95( \pm 24.329)$ & 0.534 & \\
\hline Regular & $35.56( \pm 26.592)$ & & \\
\hline \multicolumn{4}{|c|}{ Duration of vitamin D supplementation in the first year of life in months } \\
\hline $0-6$ & $39.04( \pm 26.90)$ & & -0.095 \\
\hline $7-12$ & $34.95( \pm 26.07)$ & & \\
\hline \multicolumn{4}{|c|}{ Duration of breastfeeding ${ }^{d}$} \\
\hline$<2$ weeks & $36.14( \pm 23.691)$ & 0.802 & \\
\hline$>2$ weeks $^{c}$ & $36.51( \pm 27.093)$ & & \\
\hline
\end{tabular}


course, potentially outweighing the influence of vitamin D supplementation and breastfeeding. Finally, a potential confounding between different variables cannot be ruled out. For example, a higher social status often determines a healthier life style. By recruiting control cases via friends and relatives of KD cases, an attempt was made to achieve the best possible comparison regarding socio-economic status. Due to data protection regulations, we were not able to collect additional data regarding the socio-economic status of both KD and control cases.

Another limitation relates to the simultaneous screening for several risk factors. The so-called multiple testing problem (look-elsewhere effect) describes randomly significant findings due to the large number of factors studied [35]. For this reason, our findings should be verified in future prospective studies of larger and more diverse study populations.

\section{Conclusion}

In association with KD in our study population, our results indicate protective effects of vitamin D supplementation and further reinforce breastfeeding to have a protective effect. However, these factors seem not to influence the natural course of the disease. Although the effects were small, they nevertheless underscore the overall benefit of both interventions.

\section{Additional files}

Additional file 1: Questionnaire Kawasaki Disease: ESPED (populationbased german pediatric surveillance study). (PDF $146 \mathrm{~kb}$ )

Additional file 2: Questionnaire Kawasaki Disease: Perinatal risk factors. (PDF $160 \mathrm{~kb}$ )

\section{Abbreviations \\ 95\% Cl: 95\% Confidence interval; CAA: Coronary Artery Aneurysm; e.g.: for example; ESPED: Nation-wide population-based German Pediatric Surveil- lance Study; ESR: Erythrocyte sedimentation rate; IVIG: Intravenous immunoglobulin; KD: Kawasaki Disease; OR: Odds Ratio}

\section{Acknowledgements}

Special thanks to the physicians reporting Kawasaki disease cases and to the families of Kawasaki disease children participating in this study. Additional thanks to Natalie Diffloth for her English language editing.

\section{Funding}

Funding neither played a role in design, collection, analysis, interpretation of data, nor in writing the manuscript. André Jakob has received funding for this work by a grant from the German Heart Foundation. This funding covered the fee for the population-based German Pediatric Surveillance Institution (ESPED).

\section{Availability of data and materials}

All data are collected in a pseudonymous way. The merged pseudonymous data are digitally stored and are available upon reasonable request from the corresponding author as needed.

\section{Authors' contributions}

KM.: Performed data collection, contributed to the study design, statistical analysis, drafted the manuscript and approved the final manuscript as submitted. AV.: Conducted the statistical analysis and approved the final manuscript as submitted. MH.: Contributed to study design and interpretation of the data, served on the adjudicating committee and approved the final manuscript as submitted. ES.: Contributed to data collection, to the study design and approved the final manuscript as submitted. SK: Contributed to the statistical analysis and approved the final manuscript as submitted. JH.: Contributed to data collection and approved the final manuscript as submitted. RB.: Contributed to study design and interpretation of the data, served on the adjudicating committee and approved the final manuscript as submitted. MF.: Contributed to interpretation of the data, served on the adjudicating committee and approved the final manuscript as submitted. AL:: Contributed to interpretation of the data, served on the adjudicating committee and approved the final manuscript as submitted. NH.: Contributed to study design and interpretation of the data, served on the adjudicating committee and approved the final manuscript as submitted. SU.: Contributed interpretation of the data, served on the adjudicating committee and approved the final manuscript as submitted. AJ.: Conceived and designed the study, contributed to drafting the manuscript and approved the final manuscript as submitted.

Ethics approval and consent to participate

Approval for the study was provided by the Ethics Committee of the University of Freiburg (Number of application EK Freiburg 43/11_130055), in accordance with the ethical standards of the Declaration of Helsinki. All parents of KD patients had provided written informed consent.

\section{Consent for publication}

Our study does not contain personalised data.

\section{Competing interests}

The authors have no conflicts of interest to disclose. The authors have no financial relationships relevant to this article to disclose.

\section{Publisher's Note}

Springer Nature remains neutral with regard to jurisdictional claims in published maps and institutional affiliations.

\section{Author details}

${ }^{1}$ Department of Congenital Heart Disease and Pediatric Cardiology, University Heart Center Freiburg, Mathildenstraße 1, D-79106 Freiburg, Germany. ${ }^{2}$ Institute for Medical Information Processing, Biometry and Epidemiology, Ludwig-Maximilians-University of Munich, Munich, Germany. ${ }^{3}$ Division of Pediatric Infectious Disease and Rheumatology, Center for Pediatrics and Adolescent Medicine, University Medical Center Freiburg, Freiburg, Germany. ${ }^{4}$ Department for Pediatrics, University Hospital Carl Gustav Carus of the Technische Universität Dresden, Dresden, Germany. ${ }^{5}$ Department of Pediatric Cardiology, Ludwig-Maximilians-University of Munich, Munich, Germany.

Received: 31 August 2018 Accepted: 18 February 2019

Published online: 26 February 2019

\section{References}

1. Jakob A, et al. Kawasaki disease in Germany: a prospective, populationbased study adjusted for underreporting. Pediatr Infect Dis J. 2016;35(2): 129-34.

2. Son MBF, Newburger JW. Kawasaki Disease. Pediatr Rev. 2018;39(2):78-90.

3. Makino N, et al. Descriptive epidemiology of Kawasaki disease in Japan, 2011-2012: from the results of the 22nd nationwide survey. J Epidemiol. 2015;25(3):239-45.

4. Burns JC, Glode MP. Kawasaki syndrome. Lancet. 2004;364(9433):533-44.

5. Yorifuji T, Tsukahara H, Doi H. Early childhood exposure to maternal smoking and Kawasaki Disease: A longitudinal survey in Japan. Sci Total Environ. 2018;655:141-6.

6. Mclntire DD, et al. Birth weight in relation to morbidity and mortality among newborn infants. N Engl J Med. 1999:340(16):1234-8. 
7. Sevelsted A, et al. Cesarean section and chronic immune disorders. Pediatrics. 2015;135(1):e92-8.

8. Ross AC, et al. The 2011 report on dietary reference intakes for calcium and vitamin $D$ from the Institute of Medicine: what clinicians need to know. J Clin Endocrinol Metab. 2011;96(1):53-8.

9. Colotta F, Jansson B, Bonelli F. Modulation of inflammatory and immune responses by vitamin D. J Autoimmun. 2017;85:78-97.

10. Juonala $\mathrm{M}$, et al. Childhood 25-OH vitamin D levels and carotid intimamedia thickness in adulthood: the cardiovascular risk in young Finns study. J Clin Endocrinol Metab. 2015;100(4):1469-76.

11. Alibaz-Oner F, et al. Vitamin D levels in Takayasu's arteritis and a review of the literature on Vasculitides. J Clin Lab Anal. 2016;30(5):529-33.

12. Duijts $L$, et al. Prolonged and exclusive breastfeeding reduces the risk of infectious diseases in infancy. Pediatrics. 2010;126(1):e18-25.

13. Kramer, M.S. and R. Kakuma, Optimal duration of exclusive breastfeeding Cochrane Database Syst Rev, 2012(8): p. Cd003517.

14. Yorifuji T, Tsukahara H, Doi H. Breastfeeding and Risk of Kawasaki Disease: A Nationwide Longitudinal Survey in Japan. Pediatrics. 2016;137(6).

15. von Kries R, Heinrich B, Hermann M. Pädiatrische Epidemiologie in Deutschland: Forschungsinstrument ESPED (Erhebungseinheit für seltene pädiatrische Erkrankungen in Deutschland). Monatsschrift Kinderheilkunde. 2001;149(11):1191-7.

16. Newburger JW, et al. Diagnosis, treatment, and long-term management of Kawasaki disease: a statement for health professionals from the committee on rheumatic fever, endocarditis and Kawasaki disease, council on cardiovascular disease in the young, American Heart Association. Circulation 2004;110(17):2747-71.

17. Pearce N. Analysis of matched case-control studies. BMJ. 2016;352.

18. Zhang YD, et al. Changes in 25-hydroxyvitamin D3 level and its significance in children with Kawasaki disease. Zhongguo Dang Dai Er Ke Za Zhi. 2016;18(3):211-4.

19. Stagi $S$, et al. Severe vitamin D deficiency in patients with Kawasaki disease: a potential role in the risk to develop heart vascular abnormalities? Clin Rheumatol. 2016;35(7):1865-72.

20. Haddad JG. Vitamin D--solar rays, the milky way, or both? N Engl J Med. 1992;326(18):1213-5.

21. Wabitsch M, Koletzko B, Moß A. Vitamin-D-Versorgung im SäuglingsKindes-und Jugendalter. Monatsschrift Kinderheilkunde. 2011;159(8):766-74.

22. Equils $\mathrm{O}$, et al. 1,25-Dihydroxyvitamin $\mathrm{D}$ inhibits lipopolysaccharide-induced immune activation in human endothelial cells. Clin Exp Immunol. 2006; 143(1):58-64.

23. Suzuki Y, et al. Anti-inflammatory effect of 1alpha,25-dihydroxyvitamin D(3) in human coronary arterial endothelial cells: implication for the treatment of Kawasaki disease. J Steroid Biochem Mol Biol. 2009;113(1-2):134-8.

24. Kendrick J, et al. 25-Hydroxyvitamin D deficiency is independently associated with cardiovascular disease in the Third National Health and Nutrition Examination Survey. Atherosclerosis. 2009;205(1):255-60.

25. Al Mheid I, et al. Vitamin D status is associated with arterial stiffness and vascular dysfunction in healthy humans. J Am Coll Cardiol. 2011;58(2):186-92.

26. Jun JS, Jung YK, Lee DW. Relationship between vitamin D levels and intravenous immunoglobulin resistance in Kawasaki disease. Korean J Pediatr. 2017;60(7):216-20

27. WHO and UNICEF. Global strategy for infant and young child feeding: World Health Organization; 2003.

28. Manzoni P. Clinical benefits of Lactoferrin for infants and children. J Pediatr. 2016;173(Suppl):S43-52.

29. Goldman AS, et al. Immunologic protection of the premature newborn by human milk. Semin Perinatol. 1994;18(6):495-501.

30. Kleinman RE, Walker WA. The enteromammary immune system: an important new concept in breast milk host defense. Dig Dis Sci. 1979;24(11):876-82

31. Lawrence RA, Lawrence RM. Chapter 4 - biochemistry of human Milk. In: Breastfeeding (seventh edition). Philadelphia: W.B. Saunders; 2011. p. 98-152.

32. Nishimura T, Suzue J, Kaji H. Breastfeeding reduces the severity of respiratory syncytial virus infection among young infants: a multi-center prospective study. Pediatr Int. 2009;51(6):812-6.

33. Lawrence RA, Lawrence RM. Chapter 7 - facilitating an informed decision about breastfeeding. In: Breastfeeding (seventh edition). Philadelphia: W.B. Saunders; 2011. p. 215-31.

34. Rothman K, Greenland S, Lash TL. Modern epidemiology: Lippincott Williams \& Wilkins; 2008.

35. Rupert $\mathrm{G}$ Jr. Simultaneous statistical inference: Springer Science \& Business Media; 2012.

\section{Ready to submit your research? Choose BMC and benefit from:}

- fast, convenient online submission

- thorough peer review by experienced researchers in your field

- rapid publication on acceptance

- support for research data, including large and complex data types

- gold Open Access which fosters wider collaboration and increased citations

- maximum visibility for your research: over $100 \mathrm{M}$ website views per year

At BMC, research is always in progress.

Learn more biomedcentral.com/submissions 